\title{
Global reported endophthalmitis risk following intravitreal injections of anti-VEGF: a literature review and analysis
}

This article was published in the following Dove Press journal:

Clinical Ophthalmology

2 May 2015

Number of times this article has been viewed

\author{
Douglas K Sigford' \\ Shivani Reddy' \\ Christine Mollineaux ${ }^{2}$ \\ Shlomit Schaal' \\ 'Department of Ophthalmology \\ and Visual Sciences, University \\ of Louisville, Louisville, ${ }^{2}$ Department \\ of Ophthalmology and Visual Science, \\ University of Kentucky, Lexington, \\ KY, USA
}

Purpose: To report on endophthalmitis occurrence and associated risk factors following the intravitreal injection of anti-VEGF agents based on a review of published literature.

Materials and methods: A Medline search was performed using the terms "bevacizumab" and "ranibizumab". A total of 534 English-language articles of varying design and published from 2006 to November 2013 were analyzed for endophthalmitis occurrence and contributing perioperative factors.

Results: A total of 445,503 injections were counted. There were 103 cases of postinjection endophthalmitis in 176,124 injections (0.058\%) with bevacizumab (Avastin) versus 79 cases in 269,379 injections $(0.029 \%)$ with ranibizumab (Lucentis). This difference was due to a significantly higher occurrence of culture-negative endophthalmitis associated with bevacizumab injections. Culture-positive risk was not statistically different between the two drugs. The reported use of postinjection topical antibiotics increased the risk of culture-positive endophthalmitis. No association was found with the use of povidone iodine, a lid speculum, a mask, or an operating room. Streptococcus spp. were the most prevalent causative organism, accounting for nine of $54(17 \%)$ of all culture-positive cases.

Conclusion: Reported postinjection endophthalmitis occurred significantly more in patients treated with bevacizumab than those treated with ranibizumab. However, culture-positive occurrence was similar. Despite the potential for contamination at the time of drug compounding, bevacizumab does not appear to confer a higher risk of culture-positive endophthalmitis than ranibizumab. This study also suggests antibiotic use may increase endophthalmitis occurrence.

Keywords: endophthalmitis, eye infections, intravitreal injections, panophthalmitis

\section{Introduction}

Intravitreal injections of anti-VEGF agents have become a mainstay of treatment for exudative age-related macular degeneration (exAMD), ${ }^{1}$ diabetic macular edema, ${ }^{2}$ and retinal vein occlusion. ${ }^{3}$ The relative efficacy of anti-VEGF versus alternative therapies has resulted in millions of such injections being administered annually.

Bevacizumab (Hoffmann-La Roche, Basel, Switzerland) and ranibizumab (Hoffmann-La Roche) are two of the most studied anti-VEGF medications in clinical practice, having first shown efficacy in the treatment of exAMD in $2005 .{ }^{4-6}$ These medications are frequently compared as a result of their similar mechanisms of action. Ranibizumab is a monoclonal antibody fragment directed against VEGF-A. It is US Food and Drug administration-approved for use in exAMD, diabetic macular edema, and macular edema associated with retinal vein occlusion, and is supplied directly to practices
Correspondence: Shlomit Schaal

Department of Ophthalmology and Visua Sciences, University of Louisville, 30I East Muhammad Ali Boulevard, Louisville, KY 40202, USA

Tel +l 5027514379

Fax + I 5028522737

Email s.schaal@louisville.edu 
from the manufacturer in individual vials for intraocular use. Bevacizumab is a full monoclonal antibody against VEGF-A that is widely used off-label to treat these same diseases. Because it is not provided by the manufacturer in individual doses, it is routinely aliquoted into ready-to-use syringes by compounding pharmacies or at the institution of administration. Alternatively, a single manufacturer-supplied vial may be used for a single injection only. The latter option reduces the perceived costbenefit advantage bevacizumab may hold over ranibizumab.

The most feared complication of intravitreal injections is endophthalmitis. The occurrence, ranging from roughly one case in 1,000 to one in 5,000, and characteristics of these infections are well documented, ${ }^{7-9}$ but there are still many lingering questions regarding the best preventive strategies and the sources of the causative organisms. Recent outbreaks of endophthalmitis have occurred in Miami, Nashville, Minneapolis, Los Angeles, and New York. ${ }^{10,11}$ A large, multistate outbreak of fungal meningitis occurred in 2012, prompting a recall that included ophthalmologic drugs. ${ }^{12}$

Sterile inflammation has also been linked to intraocular injections, with cases potentially occurring in clusters. ${ }^{13,14}$ The etiology of such episodes is varied, and when known may include contaminants, use of the incorrect medication, counterfeit medication, bacterial endotoxins, or temperature fluctuation. ${ }^{15,16}$

It has been postulated that the use of bevacizumab may lead to an increased incidence of postinjection endophthalmitis as a result of the different manufacturing process and extra drug manipulation that occurs with compounding. ${ }^{17}$ Because prospective data with sufficient statistical power to address small differences in risk are lacking, we report the results of a literature review through November 2013 focusing on the reported occurrence and risk factors of endophthalmitis associated with these two anti-VEGF agents.

\section{Materials and methods}

A comprehensive Medline search was performed via PubMed on November 27, 2013 using the terms "bevacizumab" and "ranibizumab". English-language articles were screened by title and abstract for relevance before all exclusion criteria were applied. Papers were identified for possible inclusion if their abstracts described intravitreal injections of bevacizumab and/or ranibizumab in any context and encompassed five or more patients. Additionally, if any exclusion criteria were satisfied in the abstract, the article was not examined. Because of the scarcity of trials comparing bevacizumab to ranibizumab directly, this was not criteria for inclusion. ${ }^{18}$ Study references were also reviewed where appropriate to uncover other potential articles.
Articles regardless of type were considered if intravitreal bevacizumab and/or ranibizumab were used in the treatment of any nonpediatric ophthalmologic condition. Exclusion criteria were treatment with a separate invasive ocular procedure within 4 weeks, including concurrent injections or procedures, injection into eyes with silicone oil, failure to report injection numbers or complications, inclusion of fewer than five patients, and the exclusion of endophthalmitis as entry criteria. Separate articles reporting on the same patients were examined carefully, including comparison of articles by study date and institution, so that injections were not counted more than once.

Information was collected regarding the number of injections, indications, operative and perioperative techniques, endophthalmitis cases and culture results, country of origin, and year published. Particular attention was paid to the use of povidone iodine, postoperative antibiotics, masks, and lid speculums, as well as to whether injections were administered in operating suites versus an office setting.

All statistical analysis was completed using $\mathrm{R}$ version 3.0.1 (R Foundation for Statistical Computing, Vienna, Austria). Statistical comparisons regarding types of studies as well as bacterial species were made with the Fisher exact test. An unadjusted relative risk (RR) and 95\% confidence interval (CI) were constructed using a generalized linear model and Poisson distribution. An adjusted RR and 95\% CI were constructed using a random-effect model using publication as a random effect as a way of adjusting for publication. Wald $P$-values were determined. A log-likelihood $P$-value is reported for the adjusted RR of endophthalmitis when comparing bevacizumab to ranibizumab. A similar analysis was done for the endophthalmitis risks (all, positive, and negative) by region (US versus Europe).

Risk of infection was calculated as number of cases of endophthalmitis relative to the number of injections. Because the percentage of endophthalmitis cases cultured was different between certain groups, a normalized risk of infection was calculated assuming the number of cultured cases reported matched the highest proportion of cases cultured in each category of analysis. No RRs were calculated for the adjusted risk of infection, because there was not enough information to distribute the additional cases over the appropriate studies.

\section{Results}

The Medline search yielded 9,517 potential articles of interest. Abstracts were reviewed, and 1,531 articles were identified for possible inclusion. After exclusion criteria were applied, 534 studies remained for analysis, of which 281 were prospective studies. No prospective studies were specifically designed to assess the risk of endophthalmitis. 
There were a total of 445,503 injections administered in the included studies, of which 176,124 (39.5\%) were bevacizumab and 269,379 (60.5\%) were ranibizumab. There was a significant difference in the types of studies reporting each drug, with 36,742 of 176,124 (20.9\%) bevacizumab injections reported prospectively versus 114,135 of 269,379 (42.4\%) ranibizumab injections $(P<0.0001$, odds ratio 0.359 , 95\% CI 0.354-0.364, Fisher's exact test). Data reported from 42 countries were included (Table S1). Indications for injections are summarized in Table 1.

There was wide variation in the reporting of perioperative protocols, with many studies omitting the exact details of the injection procedures. With extremely rare exceptions, studies did not report perioperative measures that were not

Table I Indications for intravitreal injections

\begin{tabular}{|c|c|c|c|}
\hline & Bevacizumab & Ranibizumab & Total \\
\hline exAMD & 32,153 & 82,100 & $1 \mid 4,253$ \\
\hline PED & 118 & 356 & 474 \\
\hline PCV & 1,286 & 2,885 & $4,|7|$ \\
\hline RAP & 376 & 620 & 996 \\
\hline CNVM (PD) & 32 & 0 & 32 \\
\hline CNVM (myopia) & 2,607 & $\mathrm{I}, 353$ & 3,960 \\
\hline CNVM (POHS) & 1,259 & 155 & $I, 4 \mid 4$ \\
\hline CNVM (inflammatory) & 488 & 0 & 488 \\
\hline CNVM (AS) & 330 & 329 & 659 \\
\hline CNVM (PIC) & 27 & 15 & 42 \\
\hline CNVM (MCP) & 82 & 0 & 82 \\
\hline CNVM (idiopathic) & 170 & 56 & 226 \\
\hline CNVM (various) & 112 & 203 & 315 \\
\hline DME & 5,566 & 13,109 & 18,675 \\
\hline CME (post ERM peel) & 12 & 0 & 12 \\
\hline CME (uveitic) & 146 & 26 & 172 \\
\hline CME (PCIOL) & 79 & 0 & 79 \\
\hline CME (RP) & 0 & 15 & 15 \\
\hline CRVO & 2,183 & $|, 2| 4$ & 3,397 \\
\hline BRVO & 3,622 & 2,291 & 5,913 \\
\hline RVO & I,097 & 544 & $|, 64|$ \\
\hline RAM & 16 & 0 & 16 \\
\hline PDR & 757 & 20 & 777 \\
\hline NVI (PDR) & 12 & 0 & 12 \\
\hline NVI (various) & 57 & 0 & 57 \\
\hline CSR & 101 & 72 & 173 \\
\hline FMVD & 0 & 126 & 126 \\
\hline IMT & 77 & 180 & 257 \\
\hline Radiation retinopathy & 431 & 0 & 431 \\
\hline NAION & 17 & 0 & 17 \\
\hline NVG & 0 & 71 & 71 \\
\hline Various & $|22,9| \mid$ & 163,639 & 286,550 \\
\hline
\end{tabular}

Abbreviations: exAMD, exudative age-related macular degeneration; PED, pigment epithelial detachment; PCV, polypoidal choroidal vasculopathy; RAP, retinal angiomatous proliferation; CNVM, choroidal neovascular membrane; PD, pattern dystrophy; $\mathrm{POHS}$, presumed ocular histoplasmosis syndrome; AS, angioid streaks; PIC, punctate inner choroidopathy; MCP, multifocal choroiditis and panuveitis; DME, diabetic macular edema; CME, cystoid macular edema; ERM, epiretinal membrane; PCIOL, posterior chamber intraocular lens; $R P$, retinitis pigmentosa; $C R V O$, central retinal vein occlusion; BRVO, branch RVO; RAM, retinal arterial macroaneurysm; PDR, proliferative diabetic retinopathy; $\mathrm{NVI}$, neovascularization of the iris; CSR, central serous chorioretinopathy; FMVD, foveomacular vitelliform dystrophy; IMT, idiopathic macular telangiectasia; NAION, nonarteritic ischemic optic neuropathy; NVG, neovascular glaucoma. instituted. Preinjection povidone iodine was reported in 290 studies (54.3\%), and was the most commonly reported variable perioperative condition. Postoperative antibiotic use was reported in 235 studies (44\%), lid-speculum use in 136 studies (25.5\%), operating room use in 78 studies (14.6\%), and surgical mask use for the physician in 54 studies $(10.1 \%)$. Bevacizumab and ranibizumab groups were similar in perioperative reporting (Table 2), differing only slightly in the reporting of antibiotic use $(P<0.0001$, adjusted RR 0.96 , 95\% CI 0.94-0.97).

There were 103 cases of endophthalmitis with bevacizumab injections, resulting in a risk of $0.058 \%(95 \% \mathrm{CI}$ $0.048 \%-0.071 \%)$, and there were 79 cases with ranibizumab injections for a risk of $0.029 \%$ (95\% CI $0.024 \%-0.037 \%)$. This difference was statistically significant using the randomeffect model and a $\log$-likelihood $P$-value ( $P=0.01$, adjusted RR 1.59, 95\% CI 1.11-2.29). The risk of endophthalmitis by year showed only minor variation (Figure S1). The risk was not significantly different either in retrospective versus prospective data ( $P=0.12$, adjusted RR $1.59,95 \% \mathrm{CI}$ 0.84-3.03). None of the perioperative variables had an effect on endophthalmitis risk that reached statistical significance when considering all cases of endophthalmitis together (Table 3), although this was not true when considering culture-positive and culture-negative cases separately (Tables 4 and 5). The reported use of postoperative antibiotics significantly increased the risk of culture-positive endophthalmitis ( $P=0.02$, adjusted RR 2.7, 95\% CI 1.06-7.14).

The total nonnormalized culture-positive and culture-negative occurrences of endophthalmitis of 172,991 injections in the US were 80 cases $(0.046 \%, 95 \%$ CI $0.044 \%-$ $0.058 \%), 22$ cases $(0.013 \%, 95 \%$ CI $0.009 \%-0.019 \%)$, and 55 cases $(0.032 \%, 95 \%$ CI $0.024 \%-0.041 \%)$. In Europe, of 119,477 injections, the same subgroups had 33 cases

Table 2 Percentage of papers positively reporting specific perioperative techniques

\begin{tabular}{|c|c|c|c|}
\hline & Bevacizumab & Ranibizumab & $\operatorname{RR}^{\mathrm{a}}(95 \% \mathrm{Cl})$ \\
\hline \multirow[t]{2}{*}{ Povidone iodine } & $69.00 \%$ & $52.30 \%$ & $1.00(0.99-1.01)$ \\
\hline & & & $P=0.9617$ \\
\hline \multirow[t]{2}{*}{ Antibiotics } & $50.90 \%$ & $48.00 \%$ & $0.96(0.94-0.97)$ \\
\hline & & & $P<0.000 \mid$ \\
\hline \multirow[t]{2}{*}{ Lid speculum } & $33.70 \%$ & $24.00 \%$ & $1.00(0.98-1.02)$ \\
\hline & & & $P=0.9617$ \\
\hline \multirow[t]{2}{*}{ Mask } & $6.80 \%$ & $6.30 \%$ & $1.00(0.96-1.05)$ \\
\hline & & & $P=0.9786$ \\
\hline \multirow[t]{2}{*}{ Operating room } & $7.00 \%$ & $3.80 \%$ & $1.00(0.94-1.08)$ \\
\hline & & & $P=0.9308$ \\
\hline
\end{tabular}

Notes: a Calculated with the random-effect model with Wald $P$-values comparing the reporting between drugs. Percentages are the proportion of injections for which the technique was positively reported.

Abbreviations: $\mathrm{RR}$, relative risk; $\mathrm{Cl}$, confidence interval. 
Table 3 Overall occurrence of postinjection endophthalmitis cases by perioperative technique

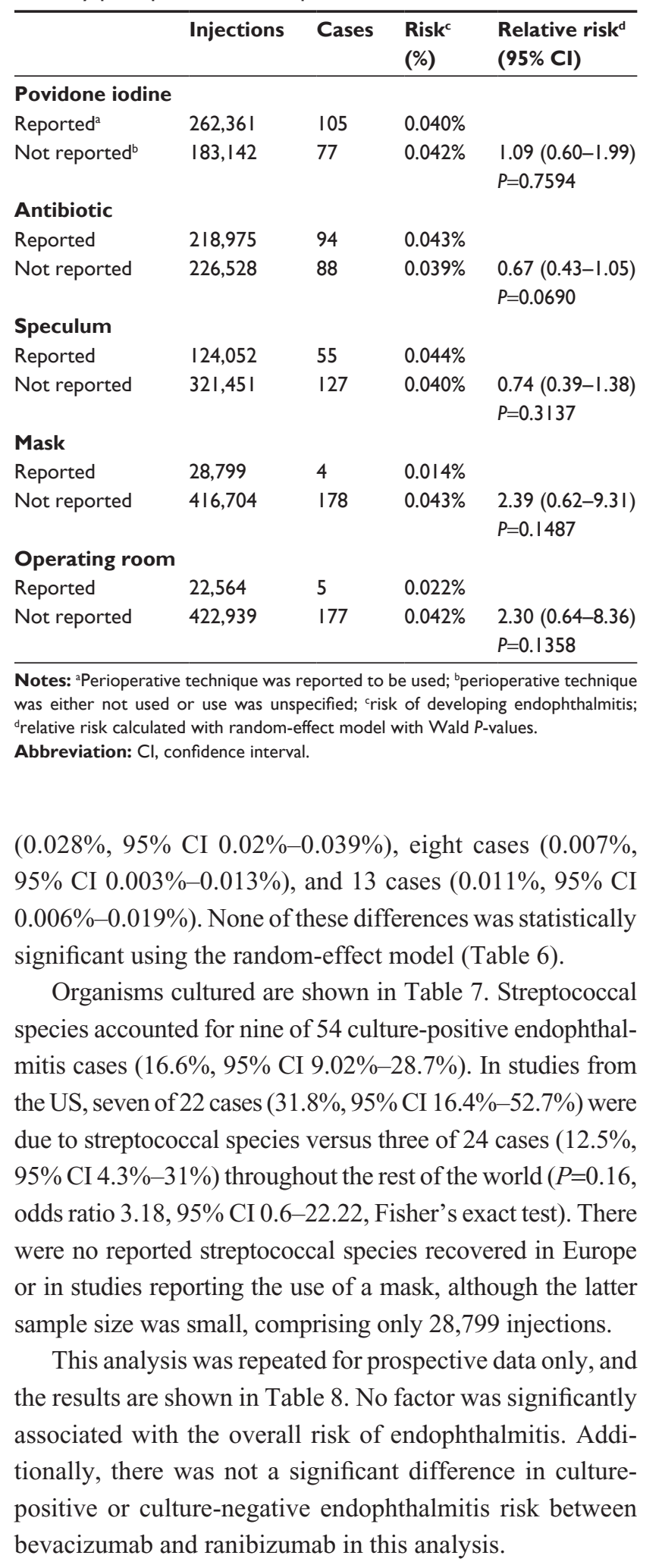

\section{Discussion}

Our understanding of the causative factors that underlie intravitreal injection-related endophthalmitis remains incomplete,
Table 4 Occurrence of culture-positive postinjection endophthalmitis cases

\begin{tabular}{|c|c|c|c|c|}
\hline & Injections & Cases & $\begin{array}{l}\text { Risk }^{c} \\
(\%)\end{array}$ & $\begin{array}{l}\text { Relative risk }^{d} \\
(95 \% \mathrm{Cl})\end{array}$ \\
\hline \multicolumn{5}{|l|}{$\overline{\text { Drug }}$} \\
\hline Ranibizumab & 269,379 & 24 & $0.011 \%$ & \\
\hline \multirow[t]{2}{*}{ Bevacizumab } & 176,124 & 30 & $0.017 \%$ & I.II (0.55-2.26) \\
\hline & & & & $P=0.7512$ \\
\hline \multicolumn{5}{|c|}{ Year of publication } \\
\hline 2013 & 181,630 & 15 & $0.008 \%$ & 1.00 (reference) \\
\hline 2012 & 67,727 & 7 & $0.013 \%$ & $2.11(0.23-19.59)$ \\
\hline 2011 & 34,684 & 3 & $0.011 \%$ & $1.98(0.16-24.00)$ \\
\hline 2010 & 41,989 & 4 & $0.010 \%$ & $2.87(0.26-31.54)$ \\
\hline 2009 & 47,268 & 8 & $0.028 \%$ & $5.11(0.63-4 I .15)$ \\
\hline 2008 & 56,636 & 15 & $0.030 \%$ & $7.88(0.94-66.01)$ \\
\hline 2007 & 3,832 & 2 & $0.066 \%$ & $14.60(1.01-209.52)$ \\
\hline \multirow[t]{2}{*}{2006} & $\mathrm{II}, 737$ & 0 & 0 & 0 \\
\hline & & & & $P=0.2556$ \\
\hline \multicolumn{5}{|l|}{ Study type } \\
\hline Prospective & $|09,54|$ & 7 & $0.010 \%$ & \\
\hline \multirow[t]{2}{*}{ Retrospective } & 335,962 & 47 & $0.014 \%$ & $2.89(0.49-16.97)$ \\
\hline & & & & $P=0.1060$ \\
\hline \multicolumn{5}{|c|}{ Povidone iodine } \\
\hline Reported $^{a}$ & 262,361 & 42 & $0.016 \%$ & \\
\hline \multirow[t]{2}{*}{ Not reported } & 183,142 & 12 & $0.007 \%$ & $0.55(0.12-2.40)$ \\
\hline & & & & $P=0.3153$ \\
\hline \multicolumn{5}{|l|}{ Antibiotic } \\
\hline Reported & 218,975 & 41 & $0.019 \%$ & \\
\hline \multirow[t]{2}{*}{ Not reported } & 226,528 & 13 & $0.006 \%$ & $0.37(0.14-0.94)$ \\
\hline & & & & $P=0.0207$ \\
\hline \multicolumn{5}{|l|}{ Speculum } \\
\hline Reported & 124,052 & 25 & $0.021 \%$ & \\
\hline \multirow[t]{2}{*}{ Not reported } & $321,45 I$ & 29 & $0.009 \%$ & 0.35 (0.09-1.39) \\
\hline & & & & $P=0.0778$ \\
\hline \multicolumn{5}{|l|}{ Mask } \\
\hline Reported & 28,799 & 0 & 0 & - \\
\hline Not reported & 416,704 & 54 & $0.013 \%$ & - \\
\hline \multicolumn{5}{|c|}{ Operating room } \\
\hline Reported & 22,564 & 0 & 0 & - \\
\hline Not reported & 422,939 & 54 & $0.013 \%$ & - \\
\hline
\end{tabular}

Notes: aPerioperative technique was reported to be used; berioperative technique was either not used or use was unspecified; 'risk of endophthalmitis adjusted for the difference in culture rates between groups; ${ }^{\circ}$ random-effect model with Wald $P$-values.

Abbreviation: $\mathrm{Cl}$, confidence interval.

despite the vast number of injections administered annually. Prospective analysis comparing the risk of endophthalmitis between different drugs or different injection techniques is problematic, because the rarity of events limits the statistical power of such studies. The purpose of the present review was to investigate these factors by combining all data published from the inception of bevacizumab and ranibizumab intraocular therapy.

There was a significantly higher risk of endophthalmitis related to bevacizumab $(0.058 \%)$ compared with ranibizumab $(0.029 \%)$. This difference was attributable to a greater risk of 
Table 5 Occurrence of culture-negative postinjection endophthalmitis cases

\begin{tabular}{|c|c|c|c|c|}
\hline & Injections & Cases & $\begin{array}{l}\text { Risk }^{\mathrm{c}} \\
\text { (\%) }\end{array}$ & $\begin{array}{l}\text { Relative risk }^{\mathrm{d}} \\
(95 \% \mathrm{Cl})\end{array}$ \\
\hline \multicolumn{5}{|l|}{ Drug } \\
\hline Ranibizumab & 269,379 & 33 & $0.015 \%$ & \\
\hline \multirow[t]{2}{*}{ Bevacizumab } & 176,124 & 64 & $0.036 \%$ & $2.12(1.26-3.57)$ \\
\hline & & & & $P=0.0026$ \\
\hline \multicolumn{5}{|c|}{ Year of publication } \\
\hline 2013 & 181,630 & 36 & $0.020 \%$ & I.00 (reference) \\
\hline 2012 & 67,727 & 17 & $0.032 \%$ & $1.16(0.16-8.16)$ \\
\hline 2011 & 34,684 & 4 & $0.014 \%$ & $0.38(0.03-5.45)$ \\
\hline 2010 & 41,989 & 25 & $0.062 \%$ & $1.45(0.15-13.66)$ \\
\hline 2009 & 47,268 & 4 & $0.014 \%$ & $0.74(0.07-7.52)$ \\
\hline 2008 & 56,636 & 6 & $0.012 \%$ & $0.81(0.07-72.63)$ \\
\hline 2007 & 3,832 & I & $0.033 \%$ & $1.83(0.05-72.63)$ \\
\hline \multirow[t]{2}{*}{2006} & 11,737 & 4 & $0.040 \%$ & $2.07(0.05-87.13)$ \\
\hline & & & & $P=0.9266$ \\
\hline \multicolumn{5}{|l|}{ Study type } \\
\hline Prospective & $|09,54|$ & 14 & $0.021 \%$ & \\
\hline \multirow[t]{2}{*}{ Retrospective } & 335,962 & 83 & $0.025 \%$ & $\mathrm{I} .89(0.4 \mathrm{I}-8.72)$ \\
\hline & & & & $P=0.2787$ \\
\hline \multicolumn{5}{|c|}{ Povidone iodine } \\
\hline Reported $^{\mathrm{a}}$ & 262,361 & 48 & $0.018 \%$ & \\
\hline \multirow[t]{2}{*}{ Not reported ${ }^{b}$} & $\mid 83,142$ & 49 & $0.029 \%$ & $1.46(0.36-5.90)$ \\
\hline & & & & $P=0.4948$ \\
\hline \multicolumn{5}{|l|}{ Antibiotic } \\
\hline Reported & 218,975 & 40 & $0.018 \%$ & \\
\hline \multirow[t]{2}{*}{ Not reported } & 226,528 & 57 & $0.027 \%$ & $0.64(0.32-1.29)$ \\
\hline & & & & $P=0.1844$ \\
\hline \multicolumn{5}{|l|}{ Speculum } \\
\hline Reported & 124,052 & 20 & $0.016 \%$ & \\
\hline \multirow[t]{2}{*}{ Not reported } & 321,451 & 77 & $0.024 \%$ & $0.84(0.19-3.82)$ \\
\hline & & & & $P=0.7806$ \\
\hline \multicolumn{5}{|l|}{ Mask } \\
\hline Reported & 28,799 & 2 & $0.012 \%$ & \\
\hline \multirow[t]{2}{*}{ Not reported } & 416,704 & 95 & $0.023 \%$ & $1.36(0.09-2.06)$ \\
\hline & & & & $P=0.0868$ \\
\hline \multicolumn{5}{|c|}{ Operating room } \\
\hline Reported & 22,564 & 1 & $0.019 \%$ & \\
\hline \multirow[t]{2}{*}{ Not reported } & 422,939 & 96 & $0.023 \%$ & $4.08(0.11-156.18$ \\
\hline & & & & $P=0.24 I 5$ \\
\hline
\end{tabular}

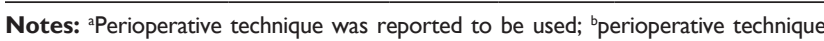
was either not used or use was unspecified; 'risk of endophthalmitis adjusted for the difference in culture rates between groups; ${ }^{d}$ random-effect model with $W$ ald $P$-values. Abbreviation: $\mathrm{Cl}$, confidence interval.

culture-negative cases in the bevacizumab group, while there was no statistically significant difference in culture-positive risk between the two groups. There was also no difference in overall, culture-positive, or culture-negative occurrence when considering prospectively reported data alone. Outbreaks of culture-positive endophthalmitis associated with bevacizumab have been reported, ${ }^{10,11}$ but the data presented here would indicate that such reports are an exception to the rule and are statistically insignificant, considering the vast amount of drugs used safely. Of course, endophthalmitis is
Table 6 Endophthalmitis risk in the US and Europe

\begin{tabular}{|c|c|c|c|}
\hline & US & Europe & $\mathbf{R R}(95 \% \mathrm{Cl})^{a}$ \\
\hline Injections & $|72,99|$ & I I9,477 & \\
\hline Endophthalmitis cases & 80 & 33 & \\
\hline \multirow[t]{2}{*}{ Risk $^{\mathrm{b}}$} & $0.046 \%$ & $0.028 \%$ & $1.25(0.44-3.52)$ \\
\hline & & & $P=0.5402$ \\
\hline Cases cultured & $96.25 \%$ & $63.64 \%$ & \\
\hline Culture-positive cases & 22 & 8 & \\
\hline \multirow[t]{2}{*}{ Culture $^{+}$risk ${ }^{\mathrm{b}}$} & $0.013 \%$ & $0.007 \%$ & $1.94(0.24-15.74)$ \\
\hline & & & $P=0.3868$ \\
\hline Normalized risk ${ }^{c}$ & $0.013 \%$ & $0.010 \%$ & - \\
\hline Culture-negative cases & 55 & 13 & \\
\hline \multirow{2}{*}{ Culture $^{-}$risk $^{b}$} & $0.032 \%$ & $0.011 \%$ & $2.05(0.44-9.49)$ \\
\hline & & & $P=0.2323$ \\
\hline Normalized risk ${ }^{c}$ & $0.032 \%$ & $0.016 \%$ & - \\
\hline
\end{tabular}

Notes: aRandom-effect model with Wald $P$-values comparing the risk of endophthalmitis between geographic areas (it is not possible to use this model with normalized risks); boccurrence of endophthalmitis; 'risk of endophthalmitis adjusted for the difference in culture rates between groups.

Abbreviations: $\mathrm{RR}$, relative risk; $\mathrm{Cl}$, confidence interval.

always significant to the patients afflicted. An alternative explanation is that an increased risk of bevacizumab-related endophthalmitis due to compounding could be offset by an increased risk of ranibizumab-related endophthalmitis at a different point in the preinjection life of the drug. It may be that drawing ranibizumab into a syringe at the time of injection may somehow increase exposure to bacteria.

Culture-negative endophthalmitis is a different issue. We found an increased risk of culture-negative endophthalmitis with bevacizumab when considering prospective and retrospective data together. Clusters of sterile endophthalmitis associated with intraocular injections of bevacizumab have

Table 7 Organisms identified from culture-positive endophthalmitis

\begin{tabular}{lll}
\hline & Bevacizumab & Ranibizumab \\
\hline Total endophthalmitis & 103 & 79 \\
Cultured & 94 & 57 \\
No growth & 64 & 33 \\
Staphylococcus epidermidis & 4 & 6 \\
Coagulase-negative Staphylococcus & 8 & 1 \\
Staphylococcus aureus & 2 & 1 \\
Total Staphylococcus spp. & 14 & 8 \\
Streptococcus viridans & 1 & 2 \\
Streptococcus salivarius & 0 & 1 \\
Streptococcus oralis & 1 & 0 \\
Streptococcus mitis & 0 & 1 \\
Streptococcus (unspecified) & 2 & 1 \\
Total Streptococcus spp. & 4 & 5 \\
Enterococcus faecalis & 1 & 0 \\
Haemophilus influenzae & $\mathrm{I}$ & 0 \\
Haemophilus (unspecified) & $\mathrm{I}$ & 0 \\
Unknown organism & 5 & 11 \\
Propionibacterium acnes & 2 & 0 \\
\hline
\end{tabular}


Table 8 Factors influencing the risk of postinjection endophthalmitis (prospective data)

\begin{tabular}{|c|c|c|c|c|}
\hline & Injections & Cases & $\begin{array}{l}\text { Risk }^{c} \\
\text { (\%) }\end{array}$ & $\begin{array}{l}\text { Relative risk }^{\mathrm{d}} \\
(95 \% \mathrm{Cl})\end{array}$ \\
\hline \multicolumn{5}{|l|}{ Drug } \\
\hline Ranibizumab & 114,135 & 51 & $0.045 \%$ & \\
\hline \multirow[t]{2}{*}{ Bevacizumab } & 36,742 & 13 & $0.035 \%$ & $0.62(0.29-1.32)$ \\
\hline & & & & $P=0.1740^{e}$ \\
\hline \multicolumn{5}{|c|}{ Year of publication } \\
\hline 2013 & 22,932 & 8 & $0.035 \%$ & 1.00 (reference) \\
\hline 2012 & 53,610 & 28 & $0.052 \%$ & $1.21(0.43-3.37)$ \\
\hline 2011 & 18,454 & 2 & $0.011 \%$ & $0.29(0.05-1.68)$ \\
\hline 2010 & 8,848 & 3 & $0.034 \%$ & $0.70(0.14-3.52)$ \\
\hline 2009 & 29,202 & 10 & $0.034 \%$ & $0.76(0.2 I-2.74)$ \\
\hline 2008 & 4,175 & 1 & $0.024 \%$ & $0.57(0.05-6.13)$ \\
\hline 2007 & $\mathrm{I}, 330$ & 1 & $0.075 \%$ & $1.97(0.19-20.17)$ \\
\hline \multirow[t]{2}{*}{2006} & 12,326 & 11 & $0.089 \%$ & $3.71(1.02-13.49)$ \\
\hline & & & & $P=0.1212^{\mathrm{e}}$ \\
\hline \multicolumn{5}{|c|}{ Povidone iodine } \\
\hline Reported ${ }^{a}$ & 47,001 & 22 & $0.047 \%$ & \\
\hline \multirow[t]{2}{*}{ Not reported } & 103,876 & 42 & $0.040 \%$ & $0.95(0.39-2.30)$ \\
\hline & & & & $P=0.8996$ \\
\hline \multicolumn{5}{|l|}{ Antibiotic } \\
\hline Reported & 42,550 & 22 & $0.052 \%$ & \\
\hline \multirow[t]{2}{*}{ Not reported } & 108,327 & 42 & $0.039 \%$ & $0.70(0.30-1.64)$ \\
\hline & & & & $P=0.4060$ \\
\hline \multicolumn{5}{|l|}{ Speculum } \\
\hline Reported & 32,173 & 16 & $0.050 \%$ & \\
\hline \multirow[t]{2}{*}{ Not reported } & 118,704 & 48 & $0.040 \%$ & $0.70(0.24-2.00)$ \\
\hline & & & & $P=0.4753$ \\
\hline \multicolumn{5}{|l|}{ Mask } \\
\hline Reported & 4,689 & 0 & 0 & - \\
\hline Not reported & $|46| 88$, & 64 & $0.044 \%$ & - \\
\hline \multicolumn{5}{|c|}{ Operating room } \\
\hline Reported & 6,585 & 0 & 0 & - \\
\hline Not reported & 144,292 & 64 & $0.044 \%$ & - \\
\hline \multicolumn{5}{|c|}{ Culture-positive cases } \\
\hline Bevacizumab & 114,135 & 0 & 0 & - \\
\hline Ranibizumab & 36,742 & 12 & $0.033 \%$ & - \\
\hline \multicolumn{5}{|c|}{ Culture-negative cases } \\
\hline Bevacizumab & 114,135 & 4 & $0.004 \%$ & \\
\hline \multirow[t]{2}{*}{ Ranibizumab } & 36,742 & 14 & $0.038 \%$ & $0.16(0.00-4.32)$ \\
\hline & & & & $P=0.1436$ \\
\hline
\end{tabular}

Notes: ${ }^{\text {PPerioperative technique was reported to be used; }{ }^{\mathrm{b}} \text { perioperative technique }}$ was either not used or use was unspecified; 'occurrence of endophthalmitis; ${ }^{d}$ randomeffect model with Wald $P$-values except where indicated; elog-likelihood comparison. Abbreviation: $\mathrm{Cl}$, confidence interval.

been reported, although the causes of these outbreaks are not always clear. Fielden et al reported multiple cases of sterile endophthalmitis associated with bevacizumab, where the lot responsible showed higher levels of endotoxin and two silicone-oil residues compared with a control sample. ${ }^{18}$ The measurements still met specifications for intravenous use however. Yamashiro et al described another cluster of cases related to a batch of bevacizumab that they speculated may have been caused by contaminating endotoxin. ${ }^{14}$ Wickremasinghe et al also reported sterile endophthalmitis with bevacizumab, and suggested a number of possible mechanisms, including endotoxins, increased immune sensitization with multiple injections, and compromised storage of the drug. ${ }^{15}$ The increased risk of culture-negative endophthalmitis with bevacizumab was not confirmed when using prospective data alone in this study.

There was no difference in overall endophthalmitis risk associated with the reporting of any perioperative factors studied, including the reported use of povidone iodine, antibiotics, a lid speculum, or an operating suite. Because omitting factors from publication is not equivalent to omitting the techniques themselves in practice, these data cannot be taken to mean that the factors are conclusively unimportant in determining the risk of endophthalmitis.

This study did not show a difference in endophthalmitis risk either, based on the reporting of mask use for the operating physician. $\mathrm{McCannel}^{19}$ suggested that there may be contamination of the surgical site or instruments by oropharyngeal flora, and Wen et al subsequently showed that speaking while above a blood agar culture plate produces more bacterial colonies than speaking while wearing a surgical mask..$^{20}$ This was also found by Simunovic et al ${ }^{21}$ and Chen et $\mathrm{a}^{22}$ who found an increase in streptococcal endophthalmitis following intravitreal injections compared with following cataract surgery. It is possible that the mask use reported does not reflect mask use in practice, leading to a discrepancy between these studies and the current study. Alternatively, this study may lack the statistical power to confirm an association with mask use and endophthalmitis, given the lack of injections given with a mask.

Culture-positive endophthalmitis was significantly increased by the reported use of antibiotics. It is impossible to say that the use of antibiotics results in a higher risk of endophthalmitis, because it is unknown how prevalent antibiotic use was in studies where it was not reported. Bhavsar et $\mathrm{al}^{23}$ with the Diabetic Retinopathy Clinical Research Network, and Cheung et $\mathrm{al}^{24}$ reported higher though not statistically different incidences of endophthalmitis in patients receiving antibiotics. Bhatt et al found no difference in endophthalmitis occurrence regardless of antibiotic use. ${ }^{25}$ It has been shown that repeated exposure to ophthalmic antibiotics selects for resistant strains of ocular and nasopharyngeal flora, ${ }^{26}$ and that drug-resistant strains may be more virulent. ${ }^{27}$

Intravitreal injections given in the US are predominantly given in the office with substerile techniques. This is in 
contrast to the practice in much of Europe, where these injections are administered in an operating room. Abell et al found that endophthalmitis occurrence in a single practice was lower for injections performed in the operating room versus those performed in an office setting. ${ }^{28}$ Because few studies reported using an operating room for injections, we used endophthalmitis occurrence in Europe versus those in the US as a surrogate. Total, culture-positive, and culturenegative endophthalmitis cases between the two regions were not statistically different using the random-effect model, with culture-positive endophthalmitis risk being nearly identical.

This study is not without flaws. As with any meta-analysis or review, the data can only be as good as the studies included. An ideal meta-analysis will include studies of good-quality, typically randomized controlled trials, which have similar inclusion and exclusion criteria and compare treatment arms directly to one another. ${ }^{29}$ Because of the scarcity of such data involving bevacizumab and ranibizumab, and the rarity of endophthalmitis, such an approach for the problem of endophthalmitis is not practical. For example, assuming an endophthalmitis risk of $0.05 \%$, one would need 100,000 injections in each group to detect a halving of this risk or 50,000 injections in each group to detect a doubling. In order to have sufficient statistical power, other types of studies must be included in an analysis of endophthalmitis. The studies included here incorporate retrospective data, heterogeneous groups, and small cohorts, all of which can introduce bias. On the other hand, endophthalmitis is somewhat unique in that there are relatively few known risk factors, unlike classic multifactorial diseases, such as AMD or coronary artery disease.

The studies included seldom reported information about how the intravitreal injections were administered. Additionally, the reporting of failure to utilize a perioperative technique is exceedingly rare. This is problematic when concluding whether certain factors alter the risk of endophthalmitis. For this reason, it is important that studies report operative techniques. It is exceedingly difficult to undertake a prospective study with 50,000-100,000 patients per arm, and thus retrospective analysis may be necessary to help answer these questions. Only English-language studies were evaluated, which also may have resulted in sampling error. We did not consider pediatric cases or papers with fewer than five patients either. It is also possible that adverse events may be reported more readily with one drug versus another. Despite the potential pitfalls, the statistical power obtained from including almost 450,000 injections may make the current analysis beneficial in suggesting modifiable factors that contribute to postinjection endophthalmitis.

\section{Conclusion}

Endophthalmitis risk was significantly higher in eyes treated with bevacizumab than with ranibizumab, because of a higher risk of culture-negative endophthalmitis in the bevacizumab group. The risk of endophthalmitis for both drugs was similar in prospective analysis. The results of this study suggest that bevacizumab may result in a slightly higher number of culture-negative endophthalmitis cases of unknown cause, but that bacterial or fungal contamination during the processing of bevacizumab likely does not significantly alter the risk of endophthalmitis when considering the vast number of injections given without incident. This study also suggests that antibiotic use postinjection may increase endophthalmitis occurrence.

\section{Acknowledgments}

Statistics were performed by Christina Pinkston, MS, biostatistician with the University of Louisville Statistical Consulting Center. The work was supported in part by an unrestricted grant from Research to Prevent Blindness, New York, NY, USA.

\section{Disclosure}

The authors report no conflicts of interest in this work.

\section{References}

1. Comparison of Age-Related Macular Degeneration Treatments Trials (CATT) Research Group, Martin DF, Maguire MG, et al. Ranibizumab and bevacizumab for treatment of neovascular age-related macular degeneration: two-year results. Ophthalmology. 2012;119: 1388-1398.

2. Diabetic Retinopathy Clinical Research Network, Elman MJ, Qin H, et al. Intravitreal ranibizumab for diabetic macular edema with prompt versus deferred laser treatment: three-year randomized trial results. Ophthalmology. 2012;119:2312-2318.

3. Heier JS, Campochiaro PA, Yau L, et al. Ranibizumab for macular edema due to retinal vein occlusions: long-term follow-up in the HORIZON trial. Ophthalmology. 2012;119:802-809.

4. Michels S, Rosenfeld PJ, Puliafito CA, Marcus EN, Venkatraman AS. Systemic bevacizumab (Avastin) therapy for neovascular age-related macular degeneration: twelve-week results of an uncontrolled open-label clinical study. Ophthalmology. 2005;112:1035-1047.

5. Rosenfeld PJ, Schwartz SD, Blumenkranz MS, et al. Maximum tolerated dose of a humanized anti-vascular endothelial growth factor antibody fragment for treating neovascular age-related macular degeneration. Ophthalmology. 2005;112:1048-1053.

6. Heier JS, Antoszyk AN, Pavan PR, et al. Ranibizumab for treatment of neovascular age-related macular degeneration: a phase I/II multicenter, controlled, multidose study. Ophthalmology. 2006;113:633.e1-e4.

7. CATT Research Group, Martin DF, Maguire MG, et al. Ranibizumab and bevacizumab for neovascular age-related macular degeneration. N Engl J Med. 2011;364:1897-1908.

8. Mason JO 3rd, White MF, Feist RM, et al. Incidence of acute onset endophthalmitis following intravitreal bevacizumab (Avastin) injection. Retina. 2008;28:564-567. 
9. Fintak DR, Shah GK, Blinder KJ, et al. Incidence of endophthalmitis related to intravitreal injection of bevacizumab and ranibizumab. Retina. 2008;28:1395-1399.

10. Gonzalez S, Rosenfeld PJ, Stewart MW, Brown J, Murphy SP. Avastin doesn't blind people, people blind people. Am J Ophthalmol. 2012; 153:196-203. e1.

11. Sheyman AT, Cohen BZ, Friedman AH, Ackert JM. An outbreak of fungal endophthalmitis after intravitreal injection of compounded combined bevacizumab and triamcinolone. JAMA Ophthalmol. 2013;131: 864-869.

12. Centers for Disease Control and Prevention. CDC responds to multistate outbreak of fungal meningitis and other infections. 2012. Available from: http://www.cdc.gov/HAI/outbreaks/currentsituation. Accessed July 30, 2013.

13. Alan R, Berger SS. Severe intraocular inflammation/endophthalmitis following off-label treatment with intravitreal bevacizumab. 2009. Available from: http://ophthalmologyrounds.ca/modules/contentManager/ upload/document/alwaysOnLine/ScientificUpdate_en.pdf. Accessed January 8, 2015.

14. Yamashiro K, Tsujikawa A, Miyamoto K, et al. Sterile endophthalmitis after intravitreal injection of bevacizumab obtained from a single batch. Retina. 2010;30:485-490.

15. Wickremasinghe SS, Michalova K, Gilhotra J, et al. Acute intraocular inflammation after intravitreous injections of bevacizumab for treatment of neovascular age-related macular degeneration. Ophthalmology. 2008; 115:1911-1915.

16. Wang F, Yu S, Liu K, et al. Acute intraocular inflammation caused by endotoxin after intravitreal injection of counterfeit bevacizumab in Shanghai, China. Ophthalmology. 2013;120:355-361.

17. Goldberg RA, Flynn HW Jr, Isom RF, Miller D, Gonzalez S. An outbreak of Streptococcus endophthalmitis after intravitreal injection of bevacizumab. Am J Ophthalmol. 2012;153:204-208. e1.

18. Fielden M, Nelson B, Kherani A. Acute intraocular inflammation following intravitreal injection of bevacizumab - a large cluster of cases. Acta Ophthalmol. 2011;89:e664-e665.
19. McCannel CA. Meta-analysis of endophthalmitis after intravitreal injection of anti-vascular endothelial growth factor agents: causative organisms and possible prevention strategies. Retina. 2011;31:654-661.

20. Wen JC, McCannel CA, Mochon AB, Garner OB. Bacterial dispersal associated with speech in the setting of intravitreous injections. Arch Ophthalmol. 2011;129:1551-1554.

21. Simunovic MP, Rush RB, Hunyor AP, Chang AA. Endophthalmitis following intravitreal injection versus endophthalmitis following cataract surgery: clinical features, causative organisms and post-treatment outcomes. Br J Ophthalmol. 2012;96:862-866.

22. Chen E, Lin MY, Cox J, Brown DM. Endophthalmitis after intravitreal injection: the importance of viridans streptococci. Retina. 2011;31: 1525-1533.

23. Bhavsar AR, Stockdale CR, Ferris FL 3rd, Brucker AJ, Bressler NM, Glassman AR. Update on risk of endophthalmitis after intravitreal drug injections and potential impact of elimination of topical antibiotics. Arch Ophthalmol. 2012;130:809-810.

24. Cheung CS, Wong AW, Lui A, Kertes PJ, Devenyi RG, Lam WC. Incidence of endophthalmitis and use of antibiotic prophylaxis after intravitreal injections. Ophthalmology. 2012;119:1609-1614.

25. Bhatt SS, Stepien KE, Joshi K. Prophylactic antibiotic use after intravitreal injection: effect on endophthalmitis rate. Retina. 2011;31: 2032-2036.

26. Kim SJ, Toma HS. Ophthalmic antibiotics and antimicrobial resistance: a randomized, controlled study of patients undergoing intravitreal injections. Ophthalmology. 2011;118:1358-1363.

27. Miño De Kaspar H, Hoepfner AS, Engelbert M, et al. Antibiotic resistance pattern and visual outcome in experimentally-induced Staphylococcus epidermidis endophthalmitis in a rabbit model. Ophthalmology. 2001;108:470-478.

28. Abell RG, Kerr NM, Allen P, Vote BJ. Intravitreal injections: is there benefit for a theatre setting? Br J Ophthalmol. 2012;96:1474-1478.

29. Li T, Dickersin K. Citation of previous meta-analyses on the same topic: a clue to perpetuation of incorrect methods? Ophthalmology. 2013;120: 1113-1119. 


\section{Supplementary materials}

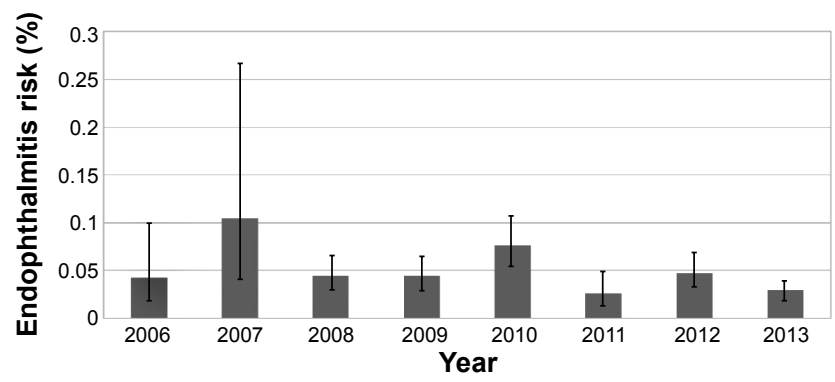

Figure SI Endophthalmitis risk by year.

Table SI Number of intravitreal injections by region/country

\begin{tabular}{|c|c|c|c|}
\hline & Bevacizumab & Ranibizumab & Total \\
\hline Asia & 0 & 979 & 979 \\
\hline Australia & 3,040 & 2,624 & 5,664 \\
\hline Austria & $3,5 \mid 4$ & 4,095 & 7,609 \\
\hline Belgium & 120 & 178 & 298 \\
\hline Bosnia and Herzegovina & 225 & 0 & 225 \\
\hline Brazil & 444 & 20 & 464 \\
\hline Canada & 8,756 & II,I88 & 19,944 \\
\hline People's Republic of China & 3,678 & 717 & 4,395 \\
\hline Croatia & 142 & 36 & 178 \\
\hline Czech Republic & 90 & 0 & 90 \\
\hline Denmark & 106 & 7,990 & 8,096 \\
\hline Egypt & 281 & 225 & 506 \\
\hline Europe & 91 & $\mathrm{I}, 70 \mathrm{I}$ & I,792 \\
\hline France & $|, 36|$ & 4,456 & 5,817 \\
\hline Germany & 5,444 & 844 & 6,288 \\
\hline Greece & $\mathrm{I}, 589$ & $\mathrm{I}, 869$ & 3,458 \\
\hline India & 1,603 & 376 & I,979 \\
\hline Iran & 6,946 & 0 & 6,946 \\
\hline Ireland & 188 & 0 & 188 \\
\hline Israel & 2,828 & 0 & 2,828 \\
\hline Italy & 2,600 & $\mathrm{I}, 348$ & 3,948 \\
\hline Japan & 5,507 & 20,840 & 26,347 \\
\hline Jordan & 60 & 0 & 60 \\
\hline Lebanon & $\mathrm{I}, 534$ & 179 & I,713 \\
\hline Mexico & 0 & 91 & 91 \\
\hline Nepal & 196 & 0 & 196 \\
\hline Netherlands & $\mathrm{I}, 794$ & 0 & I,794 \\
\hline North America & 12,585 & 14,320 & 26,905 \\
\hline Norway & 0 & 65 & 65 \\
\hline
\end{tabular}

Clinical Ophthalmology

\section{Publish your work in this journal}

Clinical Ophthalmology is an international, peer-reviewed journal covering all subspecialties within ophthalmology. Key topics include: Optometry; Visual science; Pharmacology and drug therapy in eye diseases; Basic Sciences; Primary and Secondary eye care; Patient Safety and Quality of Care Improvements. This journal is indexed on
Table SI (Continued)

\begin{tabular}{llll}
\hline & Bevacizumab & Ranibizumab & Total \\
\hline Oman & 17 & 0 & 17 \\
Poland & 232 & 162 & 394 \\
Portugal & 503 & 662 & 1,165 \\
Saudi Arabia & 11 & 0 & 11 \\
Singapore & 29 & 183 & 212 \\
Slovenia & 760 & 0 & 760 \\
South Korea & 6,223 & 2,213 & 8,436 \\
Spain & 2,739 & 6,586 & 9,325 \\
Sweden & 538 & 1,665 & 2,203 \\
Switzerland & 3,807 & 41,873 & 45,680 \\
Taiwan & 746 & 100 & 846 \\
Thailand & 29 & 76 & 105 \\
Tunisia & 267 & 0 & 267 \\
Turkey & 5,808 & 626 & 6,434 \\
UK & 4,688 & 8,982 & 13,670 \\
US & 78,819 & 94,172 & 172,991 \\
Multiple & 6,186 & 37,938 & 44,124 \\
\hline N & &
\end{tabular}

Notes: Multicenter trials involving countries from a single continent are grouped by region. Multicenter trials involving countries from multiple continents are grouped under "Multiple".

\footnotetext{
Submit your manuscript here: http://www.dovepress.com/clinical-ophthalmology-journal
}

PubMed Central and CAS, and is the official journal of The Society of Clinical Ophthalmology (SCO). The manuscript management system is completely online and includes a very quick and fair peer-review system, which is all easy to use. Visit http://www.dovepress.com/ testimonials.php to read real quotes from published authors. 